\title{
${ }^{18}$ F-FDG PET-Guided Salvage Neoadjuvant Radiochemotherapy of Adenocarcinoma of the Esophagogastric Junction: The MUNICON II Trial
}

\author{
Christian Meyer zum Büschenfelde*1,2, Ken Herrmann*3, Tibor Schuster ${ }^{4}$, Hans Geinitz $^{5}$, Rupert Langer ${ }^{6}$, Karin Becker ${ }^{6}$, \\ Katja Ott ${ }^{7}$, Matthias Ebert ${ }^{8}$, Frank Zimmermann ${ }^{5,9}$, Helmut Friess ${ }^{3}$, Markus Schwaiger ${ }^{2}$, Christian Peschel $^{1}$, \\ Florian Lordick ${ }^{1,10 \dagger}$, and Bernd Joachim Krause ${ }^{\dagger 3}$ \\ 13rd Department of Medicine (Haematology and Medical Oncology), Klinikum rechts der Isar, Technische Universität München, \\ Munich, Germany; ${ }^{2}$ Department of Surgery, Klinikum rechts der Isar, Technische Universität München, Munich, Germany; \\ ${ }^{3}$ Department of Nuclear Medicine, Klinikum rechts der Isar, Technische Universität München, Munich, Germany; ${ }^{4}$ Institute of \\ Medical Statistics and Epidemiology, Klinikum rechts der Isar, Technische Universität München, Munich, Germany; ${ }^{5}$ Department of \\ Radiation Oncology, Klinikum rechts der Isar, Technische Universität München, Munich, Germany; ${ }^{6}$ Department of Pathology, \\ Klinikum rechts der Isar, Technische Universität München, Munich, Germany; ${ }^{7}$ Department of Surgery, University of Heidelberg, \\ Heidelberg, Germany; ${ }^{8}$ nd Department of Medicine, Klinikum rechts der Isar, Technische Universität München, Munich, Germany; \\ ${ }^{9}$ Radioonkology Universitätsspital Basel, Basel, Switzerland; and ${ }^{10} 3$ rd Department of Medicine (Hematology and Medical \\ Oncology), Clinic Braunschweig and Hannover Medical School, Braunschweig, Germany
}

Previous studies demonstrated that chemotherapy-induced changes in tumor glucose metabolism measured with ${ }^{18} \mathrm{~F}-\mathrm{FDG}$ PET identify patients who benefit from preoperative chemotherapy and those who do not. The prognosis for chemotherapy metabolic nonresponders is poorer than for metabolic responders. Therefore, we initiated this prospective trial to improve the clinical outcome of metabolic nonresponders using a salvage neoadjuvant radiochemotherapy. Methods: Fifty-six patients with locally advanced adenocarcinomas of the esophagogastric junction were included. Tumor glucose uptake was assessed by ${ }^{18}$ F-FDG PET before chemotherapy and $14 \mathrm{~d}$ after initiation of chemotherapy. PET nonresponders received salvage neoadjuvant radiochemotherapy, whereas metabolic responders received neoadjuvant chemotherapy for 3 mo before surgery. Results: Thirty-three patients were metabolic responders, and 23 were nonresponders. Resection was performed on 54 patients. R0 resection rate was $82 \%$ (95\% confidence interval [Cl], 66\%-91\%) in metabolic responders and $70 \%(95 \% \mathrm{Cl}$, $49 \%-84 \%)$ in metabolic nonresponders $(P=0.51)$. Major histologic remissions were observed in 12 metabolic responders (36\%; 95\% Cl, 22\%-53\%) and 6 nonresponders $(26 \%$; $95 \% \mathrm{Cl}$, $13 \%-46 \%)$. One-year progression-free rate was $74 \% \pm 8 \%$ in PET responders and $57 \% \pm 10 \%$ in metabolic nonresponders (log rank test, $P=0.035$ ). One-year overall survival was comparable between the groups $(\sim 80 \%)$, and 2 -y overall survival was estimated to be $71 \% \pm 8 \%$ in metabolic responders and $42 \% \pm 11 \%$ in PET nonresponders (hazard ratio, $1.9 ; 95 \% \mathrm{Cl}$, $0.87-4.24 ; P=0.10$ ). Conclusion: This prospective study showed the feasibility of a PET-guided treatment algorithm.

Received Nov. 23, 2010; revision accepted May 6, 2011.

For correspondence or reprints contact: Christian Meyer zum Büschenfelde, Klinikum rechts der Isar, Technische Universität München, Ismaninger Strasse 22, D 81675 Munich, Germany.

E-mail: christian.mzb@Irz.tum.de

${ }^{*}$ Contributed equally as first coauthors to this work.

${ }^{\dagger}$ Contributed equally as senior coauthors to this work.

COPYRIGHT @ 2011 by the Society of Nuclear Medicine, Inc.
However, by comparing the groups of nonresponding patients in the current trial and the previous published MUNICON (Metabolic response evalUatioN for Individualisation of neoadjuvant Chemotherapy in Esophageal and esophagogastric adeNocarcinoma) I trial, increased histopathologic response was observed after salvage radiochemotherapy, but the primary endpoint of the study to increase the $\mathrm{R} 0$ resection rate was not met. The prognosis of the subgroup of PET nonresponders remains poor, indicating their different tumor biology.

Key Words: oncology; PET; esophageal cancer; predictive value; response

J Nucl Med 2011; 52:1189-1196

DOI: 10.2967/jnumed.110.085803

$\mathbf{P}$ atients with locally advanced adenocarcinomas of the esophagus or esophagogastric junction (AEG) are frequently offered preoperative treatment using chemotherapy alone or radiochemotherapy $(1-4)$. One rationale for giving neoadjuvant treatment is preoperative downsizing of the tumor resulting in higher $\mathrm{R} 0$ resection rates. Indeed, several randomized trials showed significantly higher $\mathrm{R} 0$ resection rates after neoadjuvant chemotherapy than with surgery alone $(1,5,6)$, and overall survival (OS) was significantly increased by the use of preoperative therapy (3-5). Therefore, neoadjuvant treatment is now widely accepted as a standard of care for locally advanced disease, but there is still debate whether radiochemotherapy or chemotherapy should be the preferred approach for patients presenting with locally advanced adenocarcinomas of the AEG (7).

Neoadjuvant chemotherapy is only beneficial in a subgroup of patients (8). A maximum of $40 \%-50 \%$ of the patients respond to standard chemotherapy or radiochemotherapy 
regimens $(9,10)$. Because patients who do not respond to chemotherapy may be compromised by toxic side effects and the delay caused by an ineffective therapy, it is desirable to have a diagnostic test for early prediction of nonresponse in order to individualize - that is, change the treatment regimen-in these patients. Unfortunately, so far no robust pretherapeutically available molecular marker predicting response or prognosis is available for esophageal and gastroesophageal cancer (11).

${ }^{18} \mathrm{~F}-\mathrm{FDG}$ PET/CT has shown promising results in assessing early therapy response and tumor control as well as prognosis in AEG $(12,13)$. Recently, our group presented the results of the MUNICON (Metabolic response evalUatioN for Individualisation of neoadjuvant Chemotherapy in Esophageal and esophagogastric adeNocarcinoma) trial (14). In this prospective, nonrandomized clinical trial, patients without metabolic response in PET performed 2 wk after the start of induction chemotherapy were taken off chemotherapy and underwent early tumor resection. In contrast, patients demonstrating a metabolic response received a full course (up to $12 \mathrm{wk}$ ) of preoperative chemotherapy. Even though the OS of metabolic nonresponders was significantly lower than that of PET responders, it was still higher than that of a historical control undergoing 3 mo of ineffective chemotherapy (12). To explore this further, we initiated the MUNICON II trial, in which we investigated whether early PET nonresponders can benefit from a preoperative salvage neoadjuvant radiochemotherapy. For this purpose we assessed R0 resection rate, histopathologic response, event-free survival, and OS and compared the parameters with ones from metabolic responders who continued chemotherapy before surgery.

\section{MATERIALS AND METHODS}

\section{Patient Characteristics}

Inclusion criteria in this study were identical to the previously published MUNICON I trial (14), in which patients with locally advanced AEG type I (distal esophageal adenocarcinoma) or type II (gastric cardia cancer) according to classification of Siewert and Stein were selected (15). Eligibility criteria comprised cT3/4 staging based on CT and endoscopic ultrasound, exclusion of distant metastases by PET, no known medical contraindications against chemotherapy with platinum plus 5-fluorouracil, or unacceptable risks for esophagectomy, as indicated by the score of Bartels et al. (16). Indication of therapy was discussed in a multidisciplinary tumor board in all patients. None of the patients had been included in the previously published studies. All participants were at least $18 \mathrm{y}$ or older and provided written informed consent.

\section{Study Design}

The MUNICON II trial was initiated as a single-center prospective nonrandomized exploratory phase II study. The protocol was approved by the local ethics committee. The study was assigned the number 2005-0041-23-19 in the European Clinical Trials Database (EudraCT) and sponsored by the Klinikum rechts der Isar, Technische Universität München. Figure 1A displays the trial design.

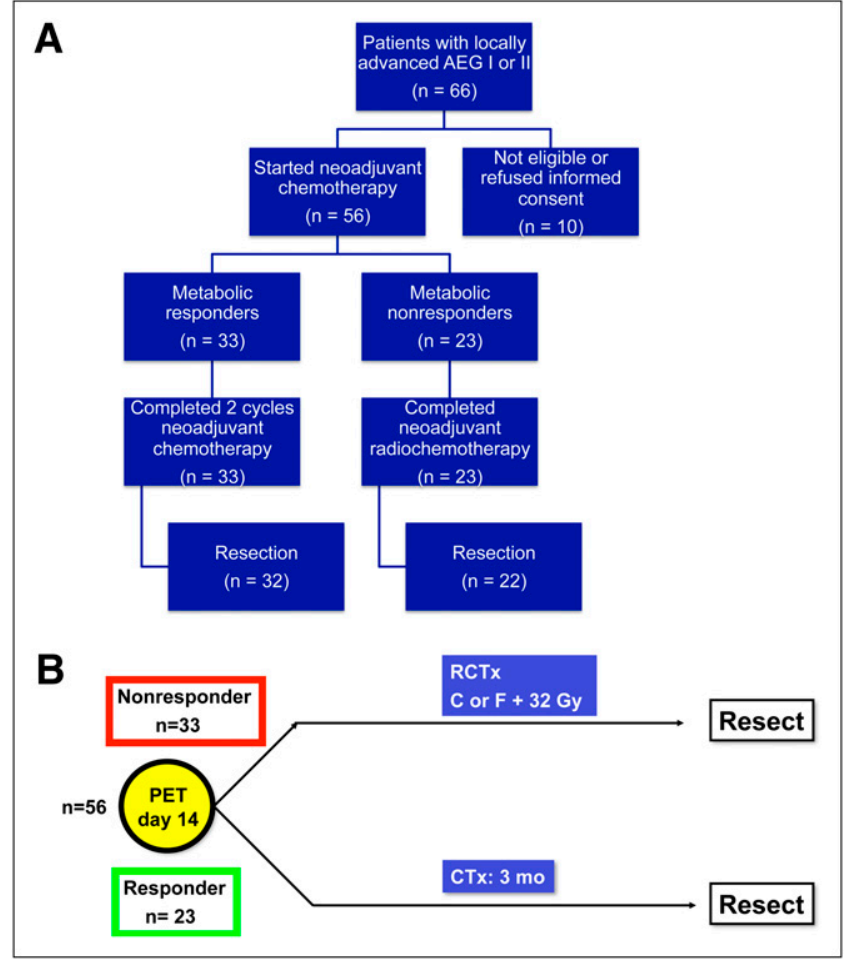

FIGURE 1. Trial profile and study design of MUNICON trial. $\mathrm{C}=$ cisplatin; $\mathrm{F}=$ 5-fluorouracil; $\mathrm{CTx}=$ chemotherapy; $\mathrm{RCTx}=$ radiochemotherapy.

According to the study protocol, metabolic response was assessed by ${ }^{18}$ F-FDG PET scans before and 2 wk after the start of chemotherapy. Metabolic responders (defined by a mean standarized uptake value [SUV] decrease of $35 \%$ or more, as previously reported $(13,14)$ ) continued chemotherapy for a maximum of 12 wk before undergoing surgery, whereas metabolic nonresponders ( $<35 \%$ mean SUV decrease) discontinued chemotherapy and proceeded to radiochemotherapy followed by surgery (Fig. 1B).

\section{Specific Procedures}

PET/CT. Patients underwent ${ }^{18} \mathrm{~F}-\mathrm{FDG}$ PET/CT scans $60 \mathrm{~min}$ after injection of approximately $370 \mathrm{MBq}$ of ${ }^{18} \mathrm{~F}-\mathrm{FDG}$ on a Sensation 16 Biograph PET/CT scanner (Siemens). A standardized ${ }^{18}$ F-FDG PET/CT protocol was used, including $6 \mathrm{~h}$ of fasting, blood glucose levels less than $150 \mathrm{mg} / \mathrm{dL}$, diluted oral contrast (Telebrix, $300 \mathrm{mg}$; Guerbet), and low-dose CT (26 mAs, $120 \mathrm{kV}$, $0.5 \mathrm{~s}$ per rotation, $5-\mathrm{mm}$ slice thickness) from the base of the skull to mid thigh for attenuation correction. Semiquantitative analysis of the PET/CT studies was performed by our standard method $(14,17)$ using a circular region of interest (ROI) (diameter, 1.5 $\mathrm{cm}$ ) with the TrueD software (Siemens Medical Solutions) and was normalized for injected dose and patients' body weight. Compared with the MUNICON I trial, all patients underwent PET on a combined PET/CT scanner. The almost simultaneous coregistered data acquisition allows a better localization and characterization of malignant lesions because of the additional anatomic information generated by the CT component. As previously shown by Souvatzoglou et al., SUVs of cancer lesions and normal organs are comparable between PET and PET/CT (18).

The mean activity administered at PET 1 was $447 \mathrm{MBq}$ of ${ }^{18} \mathrm{~F}$ FDG (median, $448 \mathrm{MBq}$; range, 341-547 MBq) and $406 \mathrm{MBq}$ of ${ }^{18} \mathrm{~F}-\mathrm{FDG}$ at PET 2 (median, $421 \mathrm{MBq}$; range, 282-535 MBq). 
The mean time interval between ${ }^{18} \mathrm{~F}-\mathrm{FDG}$ injection and start of the emission scan was $63 \mathrm{~min}$ at PET 1 (median, $61 \mathrm{~min}$; range, 55-100 $\mathrm{min}$ ) and $63 \mathrm{~min}$ at PET 2 (median, 55-77 min). The mean difference within 1 patient was $7 \mathrm{~min}$ (median, $5 \mathrm{~min}$; range, 0-42 $\mathrm{min}$ ). In the patient with a time difference of $42 \mathrm{~min}$, the PET scanner had to be restarted, causing the time delay. The second highest time differences were 22, 20, and $15 \mathrm{~min}$.

The mean blood glucose level was $99 \mathrm{mg} / \mathrm{dL}$ at PET 1 (median, $96 \mathrm{mg} / \mathrm{dL}$; range, 67-148 $\mathrm{mg} / \mathrm{dL}$ ) and $100 \mathrm{mg} / \mathrm{dL}$ at PET 2 (median, $95 \mathrm{mg} / \mathrm{dL}$; range, $67-148 \mathrm{mg} / \mathrm{dL}$ ). As no patient presented a blood glucose level above $150 \mathrm{mg} / \mathrm{dL}$, no patient had to be excluded. However, in 1 patient the initially scheduled PET/CT was rescheduled a day later because the blood glucose level was above $150 \mathrm{mg} / \mathrm{dL}$ at the first presentation.

Chemotherapy. All patients started with 2 wk of neoadjuvant chemotherapy. The mean time interval between baseline PET/CT and start of chemotherapy was $6 \mathrm{~d}$ (median, $5 \mathrm{~d}$; range, 1-34 d). The mean time interval between start of chemotherapy and second ${ }^{18}$ F-FDG PET/CT scan was $13 \mathrm{~d}$ (median, $13 \mathrm{~d}$; range, 11-19 d). Metabolic responders continued this neoadjuvant chemotherapy for a maximum of 2 cycles. Chemotherapy consisted of cisplatin (Medac $\mathrm{GmbH})\left(50 \mathrm{mg} / \mathrm{m}^{2}\right)$ given on days 1,15 , and 29 (1-h infusion time) plus folinic acid (Medac $\mathrm{GmbH})\left(500 \mathrm{mg} / \mathrm{m}^{2}\right.$ over $2 \mathrm{~h})$ and 5-fluorouracil (Hexal AG) $\left(2,000 \mathrm{mg} / \mathrm{m}^{2}\right.$ over $\left.24 \mathrm{~h}\right)$ on days $1,8,15,22,29$, and 36 , repeated on day 49 . In 10 patients with a glomerular filtration rate less than $60 \mathrm{~mL} / \mathrm{kg} / \mathrm{min}$, cisplatin was replaced by oxaliplatin (Sanofi-Aventis Group) $\left(85 \mathrm{mg} / \mathrm{m}^{2}\right.$ over $2 \mathrm{~h}$ ). In 8 patients younger than $60 \mathrm{y}$ with a good health status, paclitaxel (Bristol Myers Squibb) $\left(80 \mathrm{mg} / \mathrm{m}^{2}\right.$ over $3 \mathrm{~h}$ ) was given additionally on days 0,14 , and 28 . Adverse events were documented according to the National Cancer Institute Criteria, version 3.0.

Radiochemotherapy. After 2 wk of neoadjuvant chemotherapy, metabolic nonresponders started concurrent radiochemotherapy. Concurrent chemotherapy consisted of cisplatin $\left(6 \mathrm{mg} / \mathrm{m}^{2}\right.$ on days 1-5 and 8-12, 1-h infusion), or 5-fluorouracil $\left(250 \mathrm{mg} / \mathrm{m}^{2}\right.$, continuous infusion) if renal function did not allow cisplatin therapy. Radiation therapy was performed with photons from a linear accelerator with an energy of $6 \mathrm{MeV}$ or greater. All patients received 3 -dimensional conformal treatment planning. The planning target volume comprised the macroscopic tumor as visible on the CT and PET scans with a safety margin of $1.0-1.3 \mathrm{~cm}$. The safety margin in the craniocaudal direction along the esophagus was $4-5 \mathrm{~cm}$, to account for submucosal spread. A total dose of 32 Gy was applied at $1.6 \mathrm{~Gy} /$ fraction twice daily, 10 fractions/wk with a minimum interval of $6 \mathrm{~h}$ between the 2 daily fractions. The dose was prescribed to a reference point within the planning target volume according to International Commission on Radiation Units and Measurements 50.

Restaging. Restaging comprising endoscopy and CT was performed before and after the second cycle of chemotherapy.

Surgery. Abdominothoracic esophagectomy was performed in patients with AEG I tumors (19). Transhiatal extended gastrectomy was performed in patients with AEG II, with intraoperative frozen sections proving a proximal tumor-free resection margin. Otherwise, an abdominothoracic esophagectomy Ivor-Lewis procedure was performed in AEG II tumors. Surgery was conducted within 4-6 wk after the last administration of chemotherapy or chemoradiotherapy.

Pathology. Histopathologic response and tumor regression were assessed according to a previously published scoring system (20), differentiating between histopathologic responders $(\leq 10 \%$ residual tumor) and histopathologic nonresponders $(\geq 10 \%$ residual tumor). Involvement of the oral, aboral, and circumferential resection margins was specified according to previously published criteria (21) by 2 pathologists.

Follow-up. Patients were followed exactly as they were in the MUNICON I trial, including CT and endoscopy at 3-mo intervals during the first year after surgery. Thereafter, follow-up was performed at 6-mo intervals. Survival was calculated from the day of the second PET/CT scan. Event-free survival was calculated up to the time of death or relapse, whichever occurred first.

Statistics. According to the study protocol, R0 resection rate was the primary endpoint. On the basis of the results of MUNICON I, an improvement of R0 resection rate in metabolic nonresponders from $74 \%$ to $94 \%$ in MUNICON II was assumed. Therefore, a total of 23 PET nonresponders had to be included to detect this clinically relevant effect size at an $\alpha$-error level of 0.05 (1-sided) with a power of 0.80 . Secondary endpoints were OS, time to progression (TTP), postoperative complications and mortality, and histopathologic remission.

Differences in frequencies were analyzed using Fisher exact tests. Confidence intervals (CIs) for proportions were calculated according to the Wilson test. Survival probabilities $( \pm \mathrm{SE})$ were estimated and illustrated according to Kaplan-Meier. Statistical comparisons between different groups of patients were performed with a log-rank test, and hazard ratios were calculated using the Cox proportional hazards model. All tests were 2-sided and performed at the 5\% level of significance using PASW Statistics (version 18.0; SPSS Inc.).

\section{RESULTS}

\section{Patients}

From September 28, 2005, until November 28, 2008, 66 patients were screened. After assessment of eligibility, 10 patients proved ineligible or refused informed consent. Thus, 56 patients ( 5 women and 51 men) were treated in the study (Fig. 1A). The median age was $62 \mathrm{y}$ (range, 35-77 y). Thirty-nine patients (70\%) had AEG type I and 17 patients (30\%) had type II. Eastern Cooperative Oncology Group performance status was 0 in $77 \%$ of the patients and 1 in $23 \%$. The clinical stage as determined by endoscopic ultrasound was uT3 in 54 patients (94\%). All patients presented with clinical signs of lymph node involvement (Table 1).

\section{Chemotherapy}

Thirty-eight patients $(68 \%)$ were treated with cisplatin, folinic acid, and 5-fluorouracil; 8 (14\%) received additional paclitaxel; and $10(18 \%)$ received oxaliplatin, folinic acid, and 5-fluorouracil. For the 56 evaluable patients, cisplatin, folinic acid, and 5-fluorouracil were given to 23 responders and 15 nonresponders; oxaliplatin, folinic acid, and 5-fluorouracil were administered in 7 responders and 3 nonresponders; and paclitaxel, cisplatin, folinic acid, and 5-fluorouracil were given to 3 responders and 5 nonresponders. Frequent grade 3/4 adverse events in 56 treated patients were as follows: dysphagia, 11\%; diarrhea, $9 \%$; emesis, $2 \%$; and nausea, $2 \%$. 
TABLE 1

Baseline Characteristics of Patients Evaluated with ${ }^{18}$ F-FDG PET for Metabolic Response to Neoadjuvant Chemotherapy

\begin{tabular}{|c|c|c|c|}
\hline Characteristic & Responder $(n=33)$ & Nonresponder $(n=23)$ & $P$ \\
\hline Age (y) & & & $>0.05$ \\
\hline Median & 60 & 65 & \\
\hline Interquartile range & $35-75$ & $36-72$ & \\
\hline Sex, male $(n)$ & $30(91)$ & $21(91)$ & $>0.99$ \\
\hline Eastern Cooperative Oncology Performance Status $0(n)$ & $26(79)$ & $18(78)$ & $>0.99$ \\
\hline Localization, AEG type I (n) & $21(64)$ & $18(78)$ & 0.38 \\
\hline Intestinal type according to Laurén (n) & $23(70)$ & $17(74)$ & 0.77 \\
\hline T3 category $(n)$ & $30(91)$ & $23(100)$ & 0.26 \\
\hline $\mathrm{N}+$ category $(n)$ & $33(100)$ & $23(100)$ & $>0.99$ \\
\hline Grade G3 (n) & $20(61)$ & $11(48)$ & 0.42 \\
\hline SUV decrease (\%) & & & $<0.001$ \\
\hline Median & 51 & 19 & \\
\hline Interquartile range & $44-60$ & $5-30$ & \\
\hline
\end{tabular}

Data in parentheses are percentages, unless otherwise indicated.

\section{Radiochemotherapy}

Twenty-three PET nonresponders were referred to radiochemotherapy and received the total dose of $32 \mathrm{~Gy}$ and chemotherapy with cisplatin or 5-fluorouracil. Dysphagia was observed in $9 \%$ and nausea in $4 \%$.

\section{Metabolic Response}

After 2 wk of chemotherapy, metabolic response was assessed in 56 patients: 33 (59\%; 95\% CI, 46\%-71\%) were metabolic responders and 23 (41\%; 95\% CI, 29\%-54\%) metabolic nonresponders. There were no statistically significant differences in baseline characteristics of metabolic responders versus nonresponders with regard to age, sex, performance status, tumor localization, $\mathrm{T}$ and $\mathrm{N}$ category, or histologic subtype according to Laurén (Table 1). Metabolic response rates for patients in the different chemotherapy regimens were not significantly different (Table 2).

\section{Surgery}

Fifty-four of 56 patients (96\%) underwent surgical resection: esophagectomy in $49(90 \%)$ and transhiatal extended gastrectomy in 5 (10\%, all AEG II) patients. Because of tumor progression, 2 patients did not undergo resection (1 in each arm).

Of the 54 resected patients, 43 patients (80\%) had tumorfree resection margins (R0 resection). R0 resection (com- plete resection with no microscopic residual tumor) was achieved in 27 PET responders (82\%; 95\% CI, 66\%-91\%) and 16 PET nonresponders (70\%; 95\% CI, 49\%-84\%; $P=$ 0.51) (Table 3). Because of this lower than expected R0 resection rate in PET nonresponders, which did not exceed the presumed lower limit of interest of $74 \%$, the 1-sided primary study endpoint was not met.

The postoperative mortality rate (30-d and in-hospital mortality) was $2 \%$ (1/54 patients; $95 \%$ CI, $0.01 \%-10 \%$ ). Postoperative complications including nonsurgical morbidity were reported in $61 \%$ (20 patients; $95 \%$ CI, 44\%-75\%) of PET responders and in 70\% (16 patients; 95\% CI, 49\%$84 \%$ ) of PET nonresponders, with no statistical difference for metabolic responders versus nonresponders $(P=0.68)$ (Table 3).

\section{Pathology}

In the group of metabolic responders, 12 patients (36\%; 95\% CI, 22\%-53\%) achieved a major histopathologic response $(<10 \%$ residual tumor), whereas in the PET nonresponders, 6 patients $(26 \%$; 95\% CI, $13 \%-46 \%)$ demonstrated a histopathologic response after radiochemotherapy (Table 4). This difference was not statistically significant $(P=0.51)$. In the group of histopathologic responders, the SUV decrease was not statistically different in patients

TABLE 2

Metabolic Response Rates with Different Chemotherapy Regimens in 56 Evaluable Patients

\begin{tabular}{lrr}
\hline \multicolumn{1}{c}{ Regimen } & \multicolumn{2}{c}{ Metabolic response $(n)$} \\
\cline { 2 - 3 } & Responder $(n=33)$ & Nonresponder $(n=23)$ \\
\hline Cisplatin, folinic acid, and fluorouracil & $23(70)$ & $15(65)$ \\
Oxaliplatin, folinic acid, and fluorouracil & $7(21)$ & $3(13)$ \\
Paclitaxel, cisplatin, folinic acid, and fluorouracil & $3(9)$ & $5(22)$ \\
& & \\
\hline$n=$ number. & & \\
Differences not statistically significant. & & \\
\hline
\end{tabular}


TABLE 3

Surgical Outcome After Neoadjuvant Chemotherapy (Responders) and Neoadjuvant Radiochemotherapy (Nonresponders)

\begin{tabular}{|c|c|c|c|}
\hline \multirow[b]{2}{*}{ Regimen } & \multicolumn{2}{|c|}{ Metabolic response } & \multirow[b]{2}{*}{$P$} \\
\hline & Responder $(n=33)$ & Nonresponder $(n=23)$ & \\
\hline R0 resection & & & 0.51 \\
\hline$n$ & $27(82)$ & $16(70)$ & \\
\hline $95 \% \mathrm{Cl}$ & $66 \%-91 \%$ & $49 \%-84 \%$ & \\
\hline \multicolumn{4}{|l|}{ R1 resection } \\
\hline$n$ & $2(6)$ & $3(13)$ & \\
\hline $95 \% \mathrm{Cl}$ & $0.1 \%-20 \%$ & $3 \%-34 \%$ & \\
\hline Unknown & 4 & 4 & \\
\hline Postoperative morbidity & & & 0.68 \\
\hline$n$ & $20(61)$ & $16(70)$ & \\
\hline $95 \% \mathrm{Cl}$ & $44 \%-75 \%$ & $49 \%-84 \%$ & \\
\hline \multicolumn{4}{|l|}{ Postoperative mortality } \\
\hline$n$ & $1(3)$ & $0(0)$ & \\
\hline $95 \% \mathrm{Cl}$ & $0 \%-16 \%$ & $0 \%-15 \%$ & \\
\hline
\end{tabular}

Data in parentheses are percentages.

achieving complete histologic response (median decrease, $46 \%$; interquartile range, $30 \%-56 \%, n=5)$, compared with those with subtotal histologic remission (median decrease, $37 \%$; interquartile range, $21 \%-52 \% ; n=13 ; P=0.294$ ).

\section{Survival and TTP}

The median follow-up time was 38 mo (range, 14-54 mo). During this time, 25 patients died (12 responders and 13 nonresponders). The median OS was 38.3 mo for the whole study population and 18.3 mo for the group of PET nonresponders. For PET responders, median OS has not been reached. One-year OS was comparable between the groups
( $~ 80 \%$ ), and 2 -y OS was estimated to be $71 \% \pm 8 \%$ in metabolic responders and $42 \% \pm 11 \%$ in PET nonresponders (hazard ratio, 1.9; 95\% CI, 0.87-4.24; $P=0.10$ ) (Fig. 2A).

The median time to progression was 28 mo for the whole study population and 15.4 mo for the group of PET nonresponders. For PET responders, TTP has not been reached. One-year progression-free rate was $74 \% \pm 8 \%$ in PET responders and $57 \% \pm 10 \%$ in metabolic nonresponders. The corresponding 2-y progression-free proportion was estimated to be $64 \% \pm 9 \%$ for PET responders and $33 \% \pm$ $10 \%$ for PET nonresponders (hazard ratio, 2.22; $95 \% \mathrm{CI}$, 1.04-4.77; $P=0.035$ ) (Fig. 2B).

TABLE 4

Histopathologic Outcome After Neoadjuvant Chemotherapy (Responders) and Neoadjuvant Radiochemotherapy (Nonresponders)

\begin{tabular}{|c|c|c|c|c|}
\hline Regimen & & $\begin{array}{l}\text { Metabolic response }(n) \\
\text { Responder }(n=33)\end{array}$ & Nonresponder $(n=23)$ & $P$ \\
\hline $\begin{array}{l}\text { Regression grade } \\
\quad n \\
95 \% \mathrm{Cl}\end{array}$ & $1 \mathrm{a}$ & $\begin{array}{c}4(12) \\
3 \%-28 \%\end{array}$ & $\begin{array}{c}1(4) \\
0.1 \%-22 \%\end{array}$ & \\
\hline $\begin{array}{l}\text { Regression grade } \\
\quad n \\
95 \% \mathrm{Cl}\end{array}$ & $1 b$ & $\begin{array}{c}8(24) \\
11 \%-42 \%\end{array}$ & $\begin{array}{c}5(22) \\
7 \%-44 \%\end{array}$ & 0.56 \\
\hline $\begin{array}{l}\text { Regression grade } \\
\quad n \\
95 \% \mathrm{Cl}\end{array}$ & 2 & $\begin{array}{c}7(21) \\
9 \%-39 \%\end{array}$ & $\begin{array}{c}7(30) \\
13 \%-53 \%\end{array}$ & \\
\hline $\begin{array}{l}\text { Regression grade } \\
\quad n \\
95 \% \mathrm{Cl}\end{array}$ & 3 & $\begin{array}{c}13(39) \\
23 \%-58 \%\end{array}$ & $\begin{array}{c}9(39) \\
20 \%-61 \%\end{array}$ & \\
\hline Unknown & & 1 & 1 & \\
\hline
\end{tabular}

Data in parentheses are percentages.

Histopathologic response was scored according to Becker et al. (19): score 1a indicates complete remission, $0 \%$ residual tumor; score $1 \mathrm{~b},<10 \%$ residual tumor; score $2,10 \%-50 \%$ residual tumor; score $3,>50 \%$ residual tumor. 


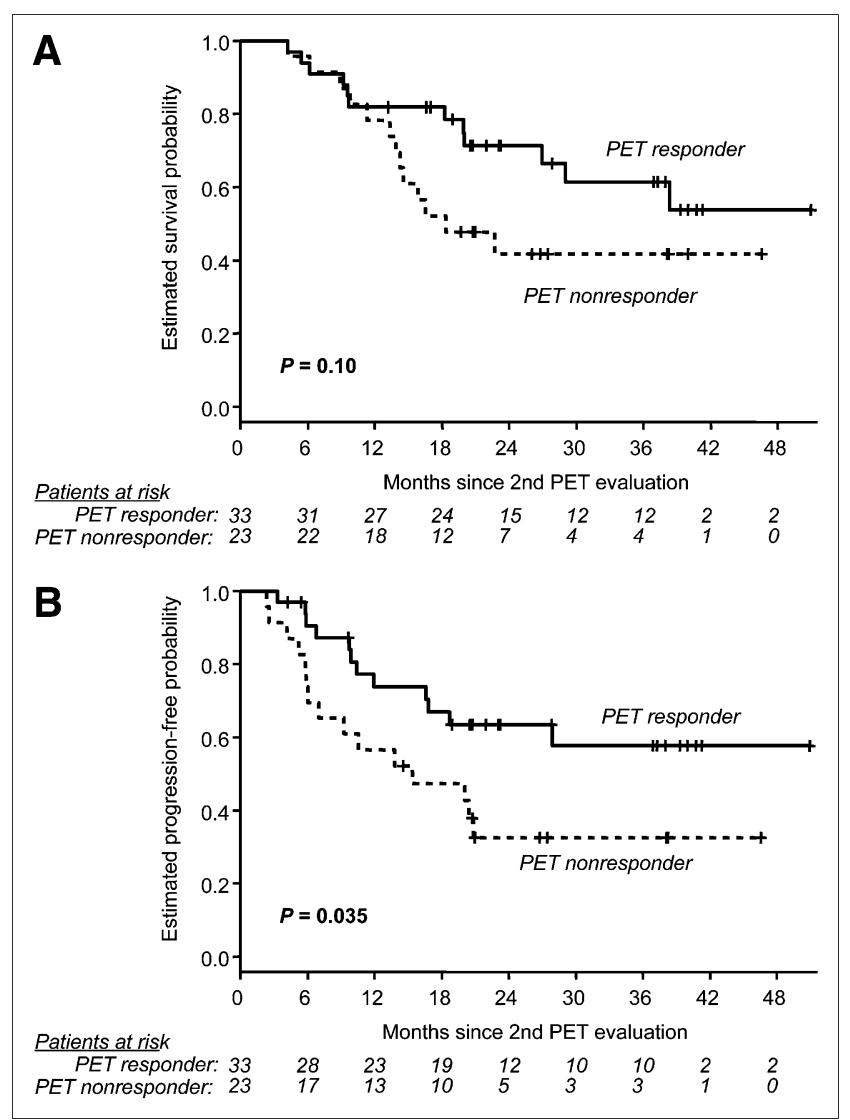

FIGURE 2. (A) OS in 56 patients assessed with PET for early metabolic response. (B) TTP in 56 patients assessed with PET for early metabolic response.

\section{Relapse}

Thirty-nine percent of PET responders and $65 \%$ of PET nonresponders displayed relapse of disease $(P=0.10)$. Interestingly, in $30 \%$ of the patients with PET-responding disease this relapse was due to distant metastases whereas $48 \%$ of PET-nonresponding patients had distant metastases $(P=0.102)$ (Table 5).

\section{DISCUSSION}

In the MUNICON II prospective clinical trial, neoadjuvant chemotherapy was switched to neoadjuvant radiochemotherapy in metabolic nonresponding patients to improve the histopathologic response, R0 resection rate, and prognosis of

TABLE 5

Relapse After Neoadjuvant Chemotherapy (Responders) and Neoadjuvant Radiochemotherapy (Nonresponders)

\begin{tabular}{lcl}
\hline Regimen & $\begin{array}{c}\text { Responder } \\
(n=33)\end{array}$ & $\begin{array}{c}\text { Nonresponder } \\
(n=23)\end{array}$ \\
\hline Relapse & $39 \%(n=13)$ & $65 \%(n=15)$ \\
Local & $9 \%(n=3)$ & $17 \%(n=4)$ \\
Distant & $30 \%(n=10)$ & $48 \%(n=11)$
\end{tabular}

Fisher exact test (relapse). patients with gastroesophageal cancer. However, although histopathologic response was observed, the primary endpoint of the study to increase the R0 resection rate was not met. Because the survival in metabolic responders (the subgroup of patients who received the same therapy in the MUNICON I and MUNICON II trials) was almost identical to the survival in the previously published MUNICON I trial, the MUNICON II trial confirms the feasibility of an early evaluation and a response-guided treatment algorithm with PET in patients with locally advanced AEG I and II.

To draw definitive conclusions, it needs to be further explored in a randomized, controlled trial whether salvage radiochemotherapy, compared with other treatment strategies in metabolically nonresponding patients, has any positive impact on outcome. To answer this question, a multicenter setting would be necessary. An international multicenter trial investigating this particular question is under way (22). Our current study was clearly a hypothesisgenerating trial, and we were trying to determine whether salvage radiochemotherapy could change the clinical course of patients receiving neoadjuvant chemotherapy who did not achieve a response. A strength of this trialand previous studies from our group-is the homogeneity of the study population with regard to histology, tumor localization, $\mathrm{T}$ and $\mathrm{N}$ stages, and type of surgery; all patients underwent standardized staging procedures and were seen by a multidisciplinary treatment team before the start of treatment. This strength is reflected by the fact that the median TTP and OS did not differ significantly across the trials. Table 6 summarizes the major findings of the MUNICON I and MUNICON II trials. Nevertheless, conclusions from historic comparisons must be drawn with caution and prospective randomized clinical trials are urgently necessary to investigate the promising results obtained in a single-center study.

As in our previous trials, tumors were assessed by a single-slice ROI. Indeed, there is debate whether singleslice ROI is the most accurate way to analyze tumor response (23). On the other hand, we recently compared different SUV-based methods for response prediction to neoadjuvant radiochemotherapy in locally advanced rectal cancer. Of note, despite the different characteristics of the ROI methods, early and late changes in SUV measured were similar regardless of the method used (24). Most importantly, our threshold and the method that we used to measure response in the present study were established and validated prospectively in previous trials. Therefore, we decided to keep this established and validated method to measure response by single-slice ROI in the MUNICON II trial.

${ }^{18} \mathrm{~F}-\mathrm{FDG}$ PET has shown promising results in assessing response to therapy and local tumor control and in prognosis (25-30). However, most of these studies investigated late response assessment after 6 or more weeks of chemotherapy and radiochemotherapy. Recently Kim et al. indicated a relatively strong concordance of $71 \%$ between 
TABLE 6

Comparison of Major Findings of MUNICON I and MUNICON II Trials

\begin{tabular}{|c|c|c|c|c|}
\hline \multirow[b]{2}{*}{ Parameter } & \multicolumn{2}{|c|}{ MUNICON I } & \multicolumn{2}{|c|}{ MUNICON II } \\
\hline & Responder & Nonresponder & Responder & Nonresponder \\
\hline PET & $49 \%$ & $51 \%$ & $59 \%$ & $41 \%$ \\
\hline \multicolumn{5}{|l|}{ Histopathologic* } \\
\hline 1 & $58 \%$ & $0 \%$ & $36 \%$ & $26 \%$ \\
\hline 2 & $20 \%$ & $4 \%$ & $21 \%$ & $30 \%$ \\
\hline 3 & $22 \%$ & $96 \%$ & $39 \%$ & $39 \%$ \\
\hline \multicolumn{5}{|l|}{ Surgery } \\
\hline Ro & $96 \%$ & $74 \%$ & $82 \%$ & $70 \%$ \\
\hline $\mathrm{R} 1$ & $4 \%$ & $26 \%$ & $6 \%$ & $13 \%$ \\
\hline$R X$ & & & $12 \%$ & $17 \%$ \\
\hline \multicolumn{5}{|l|}{ Survival } \\
\hline Median TTP & $32.9 \mathrm{mo}$ & $14.3 \mathrm{mo}$ & Not reached & $15.4 \mathrm{mo}$ \\
\hline Median OS & Not reached & $25.8 \mathrm{mo}$ & Not reached & $18.3 \mathrm{mo}$ \\
\hline
\end{tabular}

*Metabolic nonresponder, 2 wk of chemotherapy in MUNICON I vs. 2 wk of chemotherapy and 10 wk of radiochemotherapy in MUNICON II.

histopathologic complete response and metabolic complete response (31). However, the main drawback of late response assessment is that it does not allow the therapy to be modified for patients. This is in contrast to early PET response evaluation, in which PET-nonresponding patients can be offered a different, more effective therapeutic strategy. In the present study, a salvage radiochemotherapy was offered to patients not responding to chemotherapy.

Even though radiation dose was relatively low and no new radiosensitizers were applied, in the current MUNICON II trial $26 \%$ of initial metabolic nonresponders during chemotherapy had a major histopathologic response after radiochemotherapy, whereas almost no histopathologic response after radiochemotherapy was observed in PET nonresponders continuing with chemotherapy in previous trials. Thus, the chosen radiochemotherapy regimen was active enough to induce tumor cell kill in a subset of patients. Nonetheless, whereas only $9 \%$ of the PET responders undergoing chemotherapy without radiation had a local recurrence, $17 \%$ of the PET nonresponders undergoing radiochemotherapy had local treatment failure. Even more pronounced is the observation that almost $50 \%$ of the PET nonresponders had progressive disease shortly after radiochemotherapy because of distant metastases, indicating the unfavorable tumor biology that could not be reversed by radiation plus a relatively smooth concurrent chemotherapy. One reason for choosing this moderate-intensity radiochemotherapy schedule was the awareness that more aggressive regimens inevitably result in higher toxicity, lead to a higher preoperative drop-out rate, and are associated with a higher postoperative morbidity and mortality $(32,33)$. In addition to higher response rates, greater histopathologic response has been shown to be induced by neoadjuvant radiochemotherapy than by chemotherapy alone in the phase III PreOperative Chemotherapy or Radiochemotherapy in Esophago-gastric Adenocarcinoma Trial (34). However, this trial and a recent meta-analysis did not show a statistically significant survival benefit for radiochemotherapy compared with chemotherapy alone (4).

Complete tumor resection is an accepted prognostic factor in primary surgery of esophageal cancer $(19,35)$. The prognostic significance of a histopathologic response is less clearly defined, although some studies demonstrated a significant correlation with survival $(36,37)$. On the basis of the fact that radiochemotherapy results in higher histopathologic response rates, we hypothesized that salvage radiochemotherapy in chemotherapy PET nonresponders may increase the number of patients with histopathologic major remissions as well as possibly lead to a higher rate of $\mathrm{R} 0$ resections. This is the reason why we chose the $\mathrm{R} 0$ resection rate as the primary endpoint of this study. However, neoadjuvant radiochemotherapy was not able to increase the R0 resection rate in the present study, although major histopathologic remission after radiochemotherapy was observed in 6 patients $(16 \%)$.

\section{CONCLUSION}

Salvage neoadjuvant radiochemotherapy in metabolic nonresponders leads to local remissions in a considerable number of patients but was not able to change the clinical course in general because of the systemic disease. Future investigations could address whether the prognosis can be improved by adding other systemically active drugs.

\section{DISCLOSURE STATEMENT}

The costs of publication of this article were defrayed in part by the payment of page charges. Therefore, and solely to indicate this fact, this article is hereby marked "advertisement" in accordance with 18 USC section 1734.

\section{ACKNOWLEDGMENTS}

We gratefully acknowledge the assistance provided by the PET technologists and chemists and by the personnel of 
the Munich Center for Clinical Studies. This work was supported by the German Research Foundation (DFG) within the SFB Initiative 824 (collaborative research center) "Imaging for Selection, Monitoring and Individualization of Cancer Therapies" (SFB824, project B1). No other potential conflict of interest relevant to this article was reported.

\section{REFERENCES}

1. Medical Research Council Oesophageal Cancer Working Group. Surgical resection with or without preoperative chemotherapy in oesophageal cancer: a randomised controlled trial. Lancet. 2002;359:1727-1733.

2. Allum WH, Stenning SP, Bancewicz J, Clark PI, Langley RE. Long-term results of a randomized trial of surgery with or without preoperative chemotherapy in esophageal cancer. J Clin Oncol. 2009;27:5062-5067.

3. Cunningham D, Allum WH, Stenning SP, et al. Perioperative chemotherapy versus surgery alone for resectable gastroesophageal cancer. $N$ Engl $\mathrm{J} \mathrm{Med.}$ 2006;355:11-20.

4. Gebski V, Burmeister B, Smithers BM, Foo K, Zalcberg J, Simes J. Survival benefits from neoadjuvant chemoradiotherapy or chemotherapy in oesophageal carcinoma: a meta-analysis. Lancet Oncol. 2007;8:226-234.

5. Boige V, Pignon J, Saint-Aubert B, et al. Final results of a randomized trial comparing preoperative 5-fluorouracil/cisplatin to surgery alone in adenocarcinoma of the stomach and lower oesophagus: FNLCC ACCORD 07-FFCD 9703 trial. J Clin Oncol. 2007;25(200s; abstr 4512).

6. Schuhmacher C, Schlag PM, Lordick F, et al. Neoadjuvant chemotherapy versus chemotherapy alone for locally advanced adenocarcinoma of the stomach and cardia. Final results of the EORTC phase III randomized trial 40954. J Clin Oncol. 2007;25(June 20 supplement):4510.

7. Ilson DH. Cancer of the gastroesophageal junction: combined modality therapy. Surg Oncol Clin N Am. 2006;15:803-824.

8. Kelsen DP, Winter KA, Gunderson LL, et al. Long-term results of RTOG trial 8911 (USA Intergroup 113): a random assignment trial comparison of chemotherapy followed by surgery compared with surgery alone for esophageal cancer. J Clin Oncol. 2007;25:3719-3725.

9. Cunningham D, Starling N, Rao S, et al. Capecitabine and oxaliplatin for advanced esophagogastric cancer. $N$ Engl J Med. 2008;358:36-46.

10. Van Cutsem E, Moiseyenko VM, Tjulandin S, et al. Phase III study of docetaxel and cisplatin plus fluorouracil compared with cisplatin and fluorouracil as firstline therapy for advanced gastric cancer: a report of the V325 Study Group. $J$ Clin Oncol. 2006;24:4991-4997.

11. Fareed KR, Kaye P, Soomro IN, et al. Biomarkers of response to therapy in oesophago-gastric cancer. Gut. 2009;58:127-143.

12. Ott K, Weber WA, Lordick F, et al. Metabolic imaging predicts response, survival, and recurrence in adenocarcinomas of the esophagogastric junction. J Clin Oncol. 2006;24:4692-4698.

13. Weber WA, Ott K, Becker K, et al. Prediction of response to preoperative chemotherapy in adenocarcinomas of the esophagogastric junction by metabolic imaging. J Clin Oncol. 2001;19:3058-3065.

14. Lordick F, Ott K, Krause BJ, et al. PET to assess early metabolic response and to guide treatment of adenocarcinoma of the oesophagogastric junction: the MUNICON phase II trial. Lancet Oncol. 2007;8:797-805.

15. Siewert JR, Stein HJ. Classification of adenocarcinoma of the oesophagogastric junction. Br J Surg. 1998;85:1457-1459.

16. Bartels H, Stein HJ, Schomig A, Siewert JR. Risk assessment [in German]. Chirurg. 1997;68:654-661.

17. Rosenberg R, Herrmann K, Gertler R, et al. The predictive value of metabolic response to preoperative radiochemotherapy in locally advanced rectal cancer measured by PET/CT. Int J Colorectal Dis. 2009;24:191-200.

18. Souvatzoglou M, Bengel F, Busch R, et al. Attenuation correction in cardiac PET/CT with three different CT protocols: a comparison with conventional PET. Eur J Nucl Med Mol Imaging. 2007;34:1991-2000.
19. Ott K, Bader FG, Lordick F, Feith M, Bartels H, Siewert JR. Surgical factors influence the outcome after Ivor-Lewis esophagectomy with intrathoracic anastomosis for adenocarcinoma of the esophagogastric junction: a consecutive series of 240 patients at an experienced center. Ann Surg Oncol. 2009;16:1017-1025.

20. Becker K, Mueller JD, Schulmacher C, et al. Histomorphology and grading of regression in gastric carcinoma treated with neoadjuvant chemotherapy. Cancer. 2003;98:1521-1530.

21. Sarbia M, Becker KF, Hofler H. Pathology of upper gastrointestinal malignancies. Semin Oncol. 2004;31:465-475.

22. Lordick F, Ruers T, Aust DE, et al. European Organisation of Research and Treatment of Cancer (EORTC) Gastrointestinal Group. Workshop on the role of metabolic imaging in the neoadjuvant treatment of gastrointestinal cancer. Eur J Cancer. 2008;44:1807-1819.

23. Krak NC, Boellaard R, Hoekstra OS, Twisk JW, Hoekstra CJ, Lammertsma AA. Effects of ROI definition and reconstruction method on quantitative outcome and applicability in a response monitoring trial. Eur J Nucl Med Mol Imaging. 2005;32:294-301.

24. Herrmann K, Bundschuh RA, Rosenberg R. Comparison of different SUV-based methods for response prediction to neoadjuvant radiochemotherapy in locally advanced rectal cancer by FDG-PET and MRI. Mol Imaging Biol. October 9, 2010 [Epub ahead of print].

25. Downey RJ, Akhurst T, Ilson D, et al. Whole body ${ }^{18}$ FDG-PET and the response of esophageal cancer to induction therapy: results of a prospective trial. J Clin Oncol. 2003;21:428-432.

26. Flamen P, Van Cutsem E, Lerut A, et al. Positron emission tomography for assessment of the response to induction radiochemotherapy in locally advanced oesophageal cancer. Ann Oncol. 2002;13:361-368.

27. Javeri H, Xiao L, Rohren E, et al. The higher the decrease in the standardized uptake value of positron emission tomography after chemoradiation, the better the survival of patients with gastroesophageal adenocarcinoma. Cancer. 2009;115:5184-5192.

28. Levine EA, Farmer MR, Clark P, et al. Predictive value of 18-fluoro-deoxyglucose-positron emission tomography $\left({ }^{18} \mathrm{~F}\right.$-FDG-PET) in the identification of responders to chemoradiation therapy for the treatment of locally advanced esophageal cancer. Ann Surg. 2006;243:472-478.

29. Swisher SG, Erasmus J, Maish M, et al. 2-Fluoro-2-deoxy-D-glucose positron emission tomography imaging is predictive of pathologic response and survival after preoperative chemoradiation in patients with esophageal carcinoma. Cancer. 2004;101:1776-1785.

30. Vallbohmer D, Holscher AH, Dietlein $\mathrm{M}$, et al. $\left[{ }^{18} \mathrm{~F}\right]$-Fluorodeoxyglucosepositron emission tomography for the assessment of histopathologic response and prognosis after completion of neoadjuvant chemoradiation in esophageal cancer. Ann Surg. 2009;250:888-894.

31. Kim MK, Ryu JS, Kim SB, et al. Value of complete metabolic response by ${ }^{18} \mathrm{~F}$ fluorodeoxyglucose-positron emission tomography in oesophageal cancer for prediction of pathologic response and survival after preoperative chemoradiotherapy. Eur J Cancer. 2007;43:1385-1391.

32. Fiorica F, Di Bona D, Schepis F, et al. Preoperative chemoradiotherapy for oesophageal cancer: a systematic review and meta-analysis. Gut. 2004;53:925930.

33. Steyerberg EW, Neville BA, Koppert LB, et al. Surgical mortality in patients with esophageal cancer: development and validation of a simple risk score. $J$ Clin Oncol. 2006;24:4277-4284.

34. Stahl M, Walz MK, Stuschke M, et al. Phase III comparison of preoperative chemotherapy compared with chemoradiotherapy in patients with locally advanced adenocarcinoma of the esophagogastric junction. $J$ Clin Oncol. 2009;27:851-856.

35. Rizk NP, Seshan VE, Bains MS, et al. Prognostic factors after combined modality treatment of squamous cell carcinoma of the esophagus. J Thorac Oncol. 2007; 2:1117-1123.

36. Chirieac LR, Swisher SG, Ajani JA, et al. Posttherapy pathologic stage predicts survival in patients with esophageal carcinoma receiving preoperative chemoradiation. Cancer. 2005;103:1347-1355.

37. Langer R, Ott K, Feith M, Lordick F, Siewert JR, Becker K. Prognostic significance of histopathological tumor regression after neoadjuvant chemotherapy in esophageal adenocarcinomas. Mod Pathol. 2009;22:1555-1563. 